\title{
Galactic Chemical Evolution in the Context of the Recently Revealed SNe Ia Delay Time Distribution
}

\author{
Takuji Tsujimoto \\ National Astronomical Observatory of Japan, Mitaka, Tokyo 181-8588, Japan \\ email: taku.tsujimoto@nao.ac.jp
}

\begin{abstract}
The Galaxy is composed of four distinct structures, i.e., halo, bulge, and thick and thin disks, that are formed and evolved on different timescales; thus accordingly the speeds of chemical enrichment are different from one another, which is imprinted in individual stellar abundances. To decipher them, precise knowledge of the timing of the release of nucleosynthesis materials from various production sites is critical. The delay time distribution (DTD) of Type Ia supernovae (SNe Ia), recently revealed by the SNe Ia surveys of external galaxies, is incorporated into the models of chemical evolution for each structure. Here we report that the observed chemical properties for the thin and thick disks are compatible with a new SNe Ia DTD, and suggests a close chemical connection between the two in the way that the thin disk is formed from gas left after thick disk formation. This nicely explains the lack of thin disk stars with $[\mathrm{Fe} / \mathrm{H}] \lesssim-0.8$. In this new context, a top-heavy IMF for the bulge is firmly confirmed. Finally we discuss the possibility of some modification of the DTD that might be considered for the halo case.
\end{abstract}

Keywords. Galaxy: abundances, evolution — stars: abundances

Associated with the death of stars in the form of supernovae ( $\mathrm{SNe}$ ) explosions as well as asymptotic giant branch (AGB) stars, chemical enrichment by heavy elements proceeds in galaxies. Its evolutionary path is fundamentally determined by the star formation rate (SFR) as a function of time, i.e., star formation history, together with the initial mass function (IMF). The result of chemical evolution is imprinted in the present-day stellar abundance features made by long-lived stars that we observe. We see a variation in its result among different objects, i.e., galaxies or each component of a galaxy, that reflects individual unique histories.

Recent studies of the SNe Ia rate in distant and nearby galaxies imply the different form of DTD favoring a large population of young progenitors for SNe Ia (Mannucci et al. 2006; Sullivan et al. 2006; Totani et al. 2008; Maoz et al. 2010). Some authors claim that the DTD is proportional to $t_{\text {delay }}^{-1}$ for the approximate range of $0.1 \mathrm{Gyr} \leqslant t_{\text {delay }} \leqslant 10 \mathrm{Gyr}$. It implies that about $70 \%$ of SNe Ia explode with a time delay within $1 \mathrm{Gyr}$, which will have a significant impact on the chemical evolution of galaxies.

Incorporating the new SNe Ia DTD into Galactic chemical evolution model, we explore the chemical evolution of the Galaxy. Figure 1 shows the predicted $[\mathrm{Mg} / \mathrm{Fe}]$ evolution versus $[\mathrm{Fe} / \mathrm{H}]$ for the thin disk (top panel), the thick disk (middle panel), and the bulge (bottom panel), respectively. For the thin disk, chemical evolution is assumed to start from the gas remaining after the formation of thick disk. Since the thick disk formation leaves the metal-rich gas as an end product of its chemical evolution, the evolution of $[\mathrm{Mg} / \mathrm{Fe}]$ starts from $[\mathrm{Fe} / \mathrm{H}] \sim 0$. Then, $[\mathrm{Fe} / \mathrm{H}]$ and $[\mathrm{Mg} / \mathrm{Fe}]$ decreases and increases, respectively, owing to dilution by metal-poor infalling gas with a high $[\mathrm{Mg} / \mathrm{Fe}]$ ratio $(\sim+0.4)$. This reverse evolution comes to an end when the chemical enrichment by star formation exceeds the effect of gas dilution, and subsequently an usual evolutionary path appears. In the end, the overall behavior explains, in part, a large dispersion in stellar 
ages as well as in $[\mathrm{Mg} / \mathrm{Fe}]$ among the thin disk stars. In addition, it may be possible to claim that the remaining metal-rich gas after the thick disk formation results in the presence of no metal-poor thin disk stars. On the other hand, the path for super metalrich stars is calculated with the hypothesis that some regions are contaminated by the metal-rich winds from the bulge occurred several Gyr ago (Tsujimoto et al. 2010). For the bulge, the result with a flatter IMF $(x=-1.05)$ is shown by the solid curve while that with a Salpeter IMF $(x=-1.35)$ is denoted by the dashed curve.

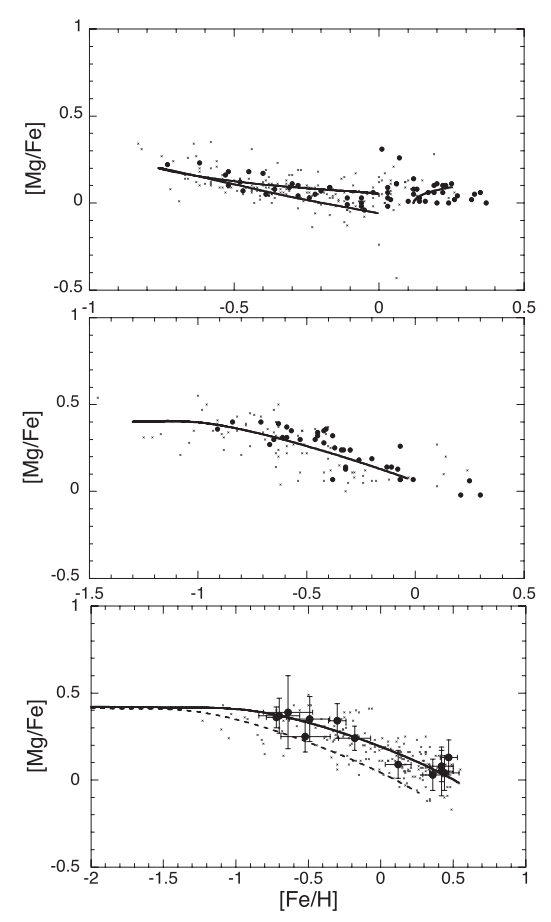

Figure 1. Chemical evolution of the thin disk (top panel), the thick disk (middle panel), and the bulge $\sim$ (bottom panel), compared with the observed quantities. In top and middle panels, the observed data are taken from Bensby et al. (2005) and Venn et al. (2004), denoted by filled circles and small crosses, respectively. In the bottom panel, the observed data are taken from Bensby et al. (2010) (filled circles) and Gonzalez et al. (2011) (small crosses).

It is a well-known fact that the Galactic halo stars have no indication of chemical enrichment from SNe Ia, considering their elemental abundances exhibit a plateau of $[\alpha / \mathrm{Fe}]$ ratio over the entire metallicity range. Thus, our understanding so far of the timescale of star formation in the halo is that it must be on the order of $10^{8} \mathrm{yr}$. More precisely to say, it was considered that halo stars were born in proto-galactic clouds or dwarf galaxies where star formation lasted for a few to several $10^{8} \mathrm{yr}$ and ended probably due to huge energy released by numerous SNe II before the initial explosion of SNe Ia. However, the new SNe Ia DTD demands its timescale within $10^{8} \mathrm{yr}$. Then, it poses a question of whether such a rapid star formation in reality occurred in the halo. Its investigation can be done by checking if the $s$-process elements, which are released from AGB stars with a timescale of $\sim 4-5 \times 10^{8} \mathrm{yr}$, are imprinted in the abundances of halo stars. Observationally, the metallicity indicating this onset among the Galactic halo stars is controversial since it is hidden by the large scatter in the abundance ratios (see Gilroy et al. 1988; Roederer et al. 2010).

\section{References}

Bensby, T., et al. 2010, A\& A, 512, A41

Bensby, T., Feltzing, S., Lundström, I., \& Ilyiin, I. 2005, $A \mathscr{E} A$, 433, 185

Gilroy, K. K., Sneden, C., Pilachowski, C. A., \& Cowan, J. J. 1988, ApJ, 327, 298

Gonzalez, O., A., et al. 2011, A\&A, 530, A54

Mannucci, F., Della Valle, M., \& Panagia, N. 2006, MNRAS, 370, 773

Maoz, D., Sharon, K., \& Gal-Yam, A. 2010, ApJ, 722, 1879

Roederer, I. U., Cowan, J. I., Karakas, A. I., Kratz, K.-L., et al. 2010, ApJ, 724, 975

Sullivan, M., et al. 2006, ApJ, 648, 868

Totani, T., Morokuma, T., Oda, T., Doi, M., \& Yasuda, N. 2008, PASJ, 60, 1327

Tsujimoto, T., Bland-Hawthorn, J., \& Freeman, K. C. 2010, PASJ, 62, 447

Venn, K. A., Irwin, M., Shetrone, M. D., Tout, C. A., Hill, V., \& Tolstoy, E. 2004, AJ, 128, 1177 